\title{
Providing lipid-based nutrient supplement during pregnancy does not reduce the risk of maternal $P$ falciparum parasitaemia and reproductive tract infections: a randomised controlled trial
}

\author{
Minyanga Nkhoma', Per Ashorn ${ }^{1,2}$, Ulla Ashorn', Kathryn G. Dewey ${ }^{3}$, Austrida Gondwe ${ }^{1}$, John Mbotwa ${ }^{4}$,
} Stephen Rogerson ${ }^{5}$, Steve M. Taylor ${ }^{6,7}$ and Kenneth Maleta ${ }^{4^{*}}$

\begin{abstract}
Background: Maternal infections are associated with maternal and foetal adverse outcomes. Nutrient supplementation during pregnancy may reduce the occurrence of infections by improving maternal immunity. We aimed to investigate the impact of small-quantity lipid-based nutrient supplement (SQ-LNS) on the occurrence of Plasmodium falciparum parasitaemia during pregnancy and trichomoniasis, vaginal candidiasis and urinary tract infection (UTI) after delivery. Methods: Pregnant Malawian women enrolled in the iLiNS-DYAD trial receiving daily supplementation with SQ-LNS, multiple micronutrients (MMN) or iron \& folic acid (IFA) from <20 gestation weeks ( $\mathrm{gw}$ ) were assessed for $P$. falciparum parasitaemia at $32 \mathrm{gw}$ using rapid diagnostic testing (RDT), at $36 \mathrm{gw}$ using polymerase chain reaction (PCR) and at delivery using both RDT and PCR; and at one week after delivery for trichomoniasis and vaginal candidiasis using wet mount microscopy and for UTI using urine dipstick analysis. The prevalence of each infection by intervention group was estimated at the prescribed time points and the global null hypothesis was tested using logistic regression. Adjusted analyses were performed using preselected covariates.

Results: The prevalence of P. falciparum parasitaemia was $10.7 \%$ at $32 \mathrm{gw}, 9 \%$ at $36 \mathrm{gw}$, and $8.3 \%$ by RDT and 20.2\% by PCR at delivery. After delivery the prevalence of trichomoniasis was $10.5 \%$, vaginal candidiasis was $0.5 \%$, and UTI was 3. $1 \%$. There were no differences between intervention groups in the prevalence of any of the infections.
\end{abstract}

Conclusion: In this population, SQ-LNS did not influence the occurrence of maternal $P$. falciparum parasitaemia, trichomoniasis, vaginal candidiasis or UTI.

Trial registration: Identifier: NCT01239693 (10 November 2010).

Keywords: Micronutrients, Pregnancy, Plasmodium falciparum, Reproductive tract infections, Urinary tract infection

\footnotetext{
* Correspondence: kmaleta@medcol.mw

${ }^{4}$ School of Public Health and Family Medicine, College of Medicine,

Mahatma Gandhi Road, Blantyre, Malawi

Full list of author information is available at the end of the article
} 


\section{Background}

Between 1990 and 2011 it was estimated that about 32\% of pregnant women attending antenatal clinics in East and Southern Africa had peripheral malaria parasitaemia [1]. Most of these infections, which are usually asymptomatic, are caused by Plasmodium falciparum and may lead to maternal anaemia [2] and low birth weight (LBW) $[3,4]$. Reproductive tract infections (RTIs) caused by Trichomonas vaginalis and Candida albicans and urinary tract infection (UTI) are also very common among pregnant women in this region and they have been associated with the occurrence of preterm birth [4-9]. LBW and preterm birth have adverse consequences for neonatal survival, subsequent childhood mortality and impaired motor and cognitive development [10-12].

Both $P$. falciparum infection and RTIs may be modified by preventive measures or presumptive treatment during pregnancy $[13,14]$. For the prevention of malaria infection in pregnancy, the World Health Organisation recommends the use of long-lasting insecticidal nets (LLINs) and in areas of stable transmission in sub-Saharan Africa, intermittent preventive treatment in pregnancy with sulphadoxine pyrimethamine (IPT-SP) [15]. While there are no recommendations for the prevention of RTIs during pregnancy, programs that screen and treat RTIs early in pregnancy have been associated with a decline in the occurrence of preterm birth and LBW [16]. Nevertheless, these prevention approaches have their own challenges including vector resistance to pyrethroid, the main insecticide used in malaria control [17]; P. falciparum resistance to SP [18]; and the risk of the development of widespread antibiotic resistance by bacterial organisms [19] if routine antibiotic use for the prevention of RTIs was adopted. For these reasons alternative methods for the prevention of maternal infections such as nutritional interventions are sought. Provision of small-quantity lipid-based nutrient supplements (SQ-LNS) is a novel nutritional intervention that supplies multiple micronutrients (MMN) and some key macronutrients such as essential fatty acids (EFAs) embedded in a lipid base [20]. When provided during

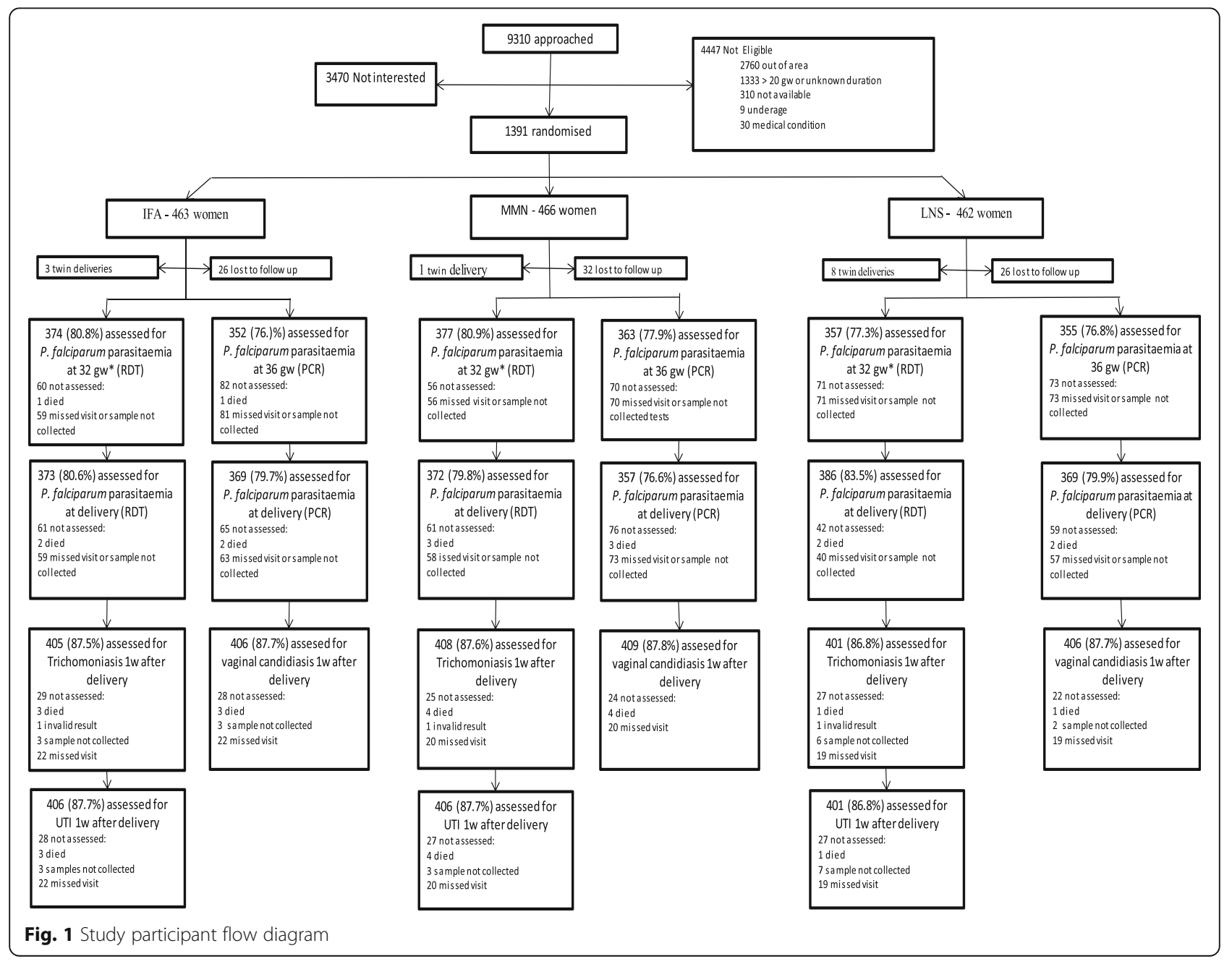


pregnancy, SQ-LNS have been shown to improve foetal growth in Ghana [21] and Bangladesh [22] and birth length in Burkina Faso [23]. However, no study has looked at the impact of antenatal provision of SQ-LNS on maternal infections.

To investigate the impact of SQ-LNS on maternal infections we measured the prevalence of maternal $P$. falciparum parasitaemia during pregnancy and at delivery and the prevalence of RTIs (trichomoniasis and vaginal candidiasis) and bacterial UTI after delivery among women in Mangochi, Malawi who were enrolled into a randomised, controlled trial that provided iron and folic acid (IFA), MMN or SQ-LNS daily [24]. We hypothesized that gestational SQ-LNS supplementation would reduce the prevalence of $P$. falciparum parasitaemia during pregnancy and the prevalence of RTIs after delivery compared to MMN and IFA. We based our assumptions on the knowledge that derivatives of the EFAs such as eicosapentaenoic acid, docosahexaenoic acid and arachidonic acid have antimalarial, antitrichomonal and antifungal properties [25-27].

\section{Methods}

The study methods have been described in detail elsewhere [24]. Briefly, this study was a sub-study of the iLiNS-DYAD-M trial, an outcome assessor-blinded randomised controlled trial that provided antenatal nutrient supplementation to improve pregnancy outcomes and child growth (trial registration: www.clinicaltrials.gov, trial identification NCT01239693). We recruited the study participants from antenatal clinics at four health facilities in Mangochi District, southern Malawi. Pregnant women were eligible to participate in the study if they were at least 15 years old, had ultrasound-confirmed pregnancy of $<20$ gestation weeks (gw), had no chronic medical condition or allergies and no evident pregnancy complications. The catchment population was rural to semi-urban and subsisted mainly on fishing and farming. Maize was the staple food in this population, which faces seasonal food insecurity with a lean period just before harvest time. Malaria transmission was holoendemic in this area.

The enrolled women were randomised into three intervention groups receiving either one tablet of IFA, the standard antenatal nutrient supplement in Malawi; one tablet of MMN which contained 18 micronutrients (including iron and folic acid) or one $20 \mathrm{~g}$ sachet of SQ-LNS containing the same 18 micronutrients and four additional minerals, EFAs (linoleic acid and $\alpha$ linolenic acid), protein, fat and $1.2 \mathrm{~g}$ of sucrose daily from enrolment to delivery [24]. The dose of iron was higher for women in the IFA group $(60 \mathrm{mg})$ than for those in the MMN and SQ-LNS groups $(20 \mathrm{mg})$. The lower iron dose in SQ-LNS was chosen so as not to greatly exceed the recommended daily intake of iron for lactating women [28], and because a dose of $20 \mathrm{mg} /$ day has in some conditions been sufficient for preventing iron deficiency anaemia in pregnancy $[20,29]$. The doses of the rest of the micronutrients in the MMN and SQ-LNS supplements were at least the recommended dietary allowance (RDA) for pregnancy, or the maximum amount that could be included in the supplement considering technical constraints [20]. The exact ingredients and dosing of the study supplements are shown in Additional file 1: Table S1. The participants also received two doses of IPT-SP according to the Malawi national guidelines at the time, which were concordant with the World Health Organisation's recommendations [30].

The outcomes reported here were pre-specified secondary outcomes measures. These were peripheral $P$. falciparum parasitaemia; trichomoniasis; vaginal candidiasis; and bacterial UTI. We diagnosed P. falciparum parasitaemia by rapid diagnostic testing (RDT) at $32 \mathrm{gw}$, by polymerase chain reaction (PCR) at $36 \mathrm{gw}$ and by RDT and PCR at delivery. RDT at 32 gw was assessed from finger prick blood samples whereas PCR at $36 \mathrm{gw}$ and RDT and PCR at delivery were assessed from venous blood samples. Study nurses performed RDT according to the manufacturer's instructions using Clearview ${ }^{\circ}$ Malaria Combo (British Biocell International Ltd., Dundee, UK) which detects the antigens histidine-rich protein 2 and Plasmodium-specific aldolase. We conducted PCR testing for P. falciparum on dried blood spot samples collected from whole blood, which were tested in a real-time PCR assay targeting the P. falciparum lactate dehydrogenase (pfldh) gene [31].

RTIs and UTIs were assessed at one week after delivery using samples from vaginal swabs and mid-stream urine, respectively. The study participant was lying in the dorsal lithotomy position for the collection of the vaginal samples. After digitally separating the labia, a study nurse advanced a cotton tipped swab through the vaginal introitus towards the posterior fornix and rotated the sample back and forth three times before pulling it out. Then the vaginal samples were sent to the laboratory where a laboratory technician processed them immediately by smearing the vaginal discharge from the swab on a microscope slide and performed wet mount microscopy after adding saline solution. We defined trichomoniasis as the visualisation of motile protozoa and candidiasis as the observation of budding yeasts, pseudohyphae or hyphal forms on microscopy. The study nurse performed urinalysis immediately after urine collection using Multistix ${ }^{\bullet} 10$ SG reagent strip (Siemens Medical Solutions USA, Inc., Pennsylvania, USA) and UTI was indicated by the presence of nitrite in a freshly voided urine sample [32]. 
A sample size of 1400 was originally calculated in accordance with the main objectives of the iLiNS-DYADM trial. This sample size provided $80 \%$ power and $95 \%$ confidence to detect an approximately 30\% reduction (among the women who received SQ-LNS, as compared to the control women) in the prevalence of maternal RTIs (from 25 to 18.3\%) or P. falciparum parasitaemia (from 15 to 9.5\%). We conducted statistical analyses using Stata 12.1 (StataCorp, College Station, USA), according to an analysis plan written and published before the intervention code was opened [24]. The analysis was based on the principle of intention-to-treat.

We estimated the prevalence of maternal $P$. falciparum parasitaemia (by intervention group) at $32 \mathrm{gw}, 36 \mathrm{gw}$ and delivery and we estimated the prevalence of the RTIs (by intervention group) at one week after delivery. We calculated Risk Ratios (RRs) for the comparison of binary end-points at a single time point and computed 95\% confidence intervals (CIs) for all the effect estimates.

We performed tests for interaction between the intervention, maternal infection and pre-specified covariates using the likelihood ratio test. The pre-specified covariates were maternal age, maternal parity, gestational age at enrolment, socio-economic score, education (completed years at school) and body-mass-index (BMI) as continuous variables; HIV status, $P$. falciparum parasitaemia, anaemia, season at enrolment and study site as categorical variables. We performed stratified analyses for all covariates that had a statistically significant interaction $(p<0.1)$.
The study protocol was approved by the College of Medicine Research and Ethics Committee, University of Malawi and the Ethics Committee of Pirkanmaa Hospital District, Finland. The trial was performed according to Good Clinical Practice guidelines and the ethical standards of Helsinki Declaration. Study staff obtained informed consent from the study participants (or their legal guardian in the case of minors) prior to performing any study procedures. An independent data safety and monitoring board monitored the incidence of suspected serious adverse events and performed interim analyses for safety.

\section{Results}

We recruited a total of 1391 pregnant women into the iLiNS-DYAD-M study between February 2011 and August 2012. The last delivery occurred in February 2013. The three groups were similar at baseline in terms of the participants' average demographic and socioeconomic characteristics and nutritional and health status $(p>0.05)$ (Table 1). The overall rate of usage of LLINs was $70.9 \%$ and was similar across the groups $(P=0.536)$. We excluded 12 women from the analysis because they had twin pregnancies.

Follow up data for $P$. falciparum parasitaemia were available from $79.6 \%$ of the women at $32 \mathrm{gw}, 76.9 \%$ at 36 gw, and from 81.4 and $78.7 \%$ at delivery by RDT and PCR respectively. Loss to follow up was similar between the groups $(P=0.805$ at $32 \mathrm{gw}, 0.638$ at $36 \mathrm{gw}, 0.180$ and 0.393 for P. falciparum parasitaemia RDT and PCR at delivery). RTI and UTI data were collected from

Table 1 Baseline characteristics of the participating women at enrolment, by intervention group

\begin{tabular}{|c|c|c|c|}
\hline Characteristic & IFA & MMN & LNS \\
\hline Number of participants & 463 & 466 & 462 \\
\hline Mean (SD) maternal age, years & $25(6)$ & $25(6)$ & $25(6)$ \\
\hline Mean (SD) maternal education, completed years & $3.9(3.4)$ & $4.1(3.4)$ & $4.1(3.6)$ \\
\hline Proportion with severely food insecure households & $34.7 \%$ & $37.5 \%$ & $35.8 \%$ \\
\hline Mean (SD) gestational age at enrolment, weeks & $16.8(2.1)$ & $16.8(2.2)$ & $16.9(2.2)$ \\
\hline Mean (SD) number of previous pregnancies & $2.1(1.8)$ & $2.1(1.8)$ & $2.2(1.7)$ \\
\hline Proportion of nulliparous women & $20.4 \%$ & $23.0 \%$ & $22.1 \%$ \\
\hline Mean (SD) height, cm & $156.1(5.7)$ & $156.0(5.6)$ & $156.1(5.7)$ \\
\hline Mean (SD) weight, kg & $53.9(7.4)$ & $54.0(8.1)$ & $54.3(8.4)$ \\
\hline Mean (SD) MUAC, cm & $26.4(2.4)$ & $26.3(2.8)$ & $26.5(2.7)$ \\
\hline Mean (SD) BMl, kg/m² & $22.1(2.6)$ & $22.2(2.9)$ & $22.2(3.0)$ \\
\hline Proportion of women with a $\mathrm{BMI}<18.5 \mathrm{~kg} / \mathrm{m}^{2}$ & $5.9 \%$ & $4.6 \%$ & $5.7 \%$ \\
\hline Mean (SD) blood haemoglobin concentration, g/l & $111(17)$ & $111(16)$ & $112(16)$ \\
\hline Proportion of anaemic women $(\mathrm{Hb}<100 \mathrm{~g} / \mathrm{l})$ & $21.0 \%$ & $19.8 \%$ & $21.2 \%$ \\
\hline Proportion of women with a positive HIV test & $15.6 \%$ & $11.1 \%$ & $14.4 \%$ \\
\hline Proportion of women with a positive P. falciparum test (RDT) & $22.7 \%$ & $24.1 \%$ & $22.8 \%$ \\
\hline
\end{tabular}

IFA iron and folic acid, LNS lipid based nutrient supplement, MMN multiple micronutrients, MUAC mid-upper arm circumference, RDT rapid diagnostic test 
$86.6 \%$ of the women. Loss to follow up was similar between the groups $(P=0.958$ for RTI and UTI after delivery) (Fig. 1). The participants who were excluded from the analysis tended to be younger (23.8 vs $25.4, P<$ 0.001 ); to be more educated (4.4 vs $3.9, P=0.025)$; to have a slightly higher BMI (22.6 vs $22.0, P=0.001$ ); to have a higher wealth index $(0.29$ vs $-0.09, P<0.001)$; to be primiparous $(31 \%$ vs $18 \%, P<0.001)$ and to be anaemic $(25 \%$ vs $19 \%, P=0.014)$.

The overall prevalence of $P$. falciparum parasitaemia was $23.3 \%$ at enrolment (RDT), $10.7 \%$ at $32 \mathrm{gw}$ (RDT); $9.0 \%$ at 36 gestation weeks (PCR); $8.3 \%$ by RDT at delivery and $20.2 \%$ by PCR at delivery. The prevalence of $P$. falciparum parasitaemia at $32 \mathrm{gw}$ (RDT), $36 \mathrm{gw}$ (PCR), delivery (RDT) and delivery (PCR) was 10.9, 7.6, 8.0 and $17.6 \%$, respectively, in the LNS group, and was similar in the IFA and MMN groups (Table 2). Relative to the IFA group, the risk for $P$. falciparum parasitaemia.at $32 \mathrm{gw}$ (RDT) was not significantly different in the SQ-LNS (RR 0.91, 95\% CI 0.6- 1.36) or MMN (RR 0.79, 95\% CI 0.521.20) groups. Similarly, when compared to MMN, the risk for P. falciparum parasitaemia at $32 \mathrm{gw}$ (RDT) in the LNS group was not significantly different (RR 1.14, 95\% CI 0.74- 1.76). The risk for $P$. falciparum parasitaemia at the other time points was also similar in all 3 study groups (Table 2 ).

The overall prevalence of trichomoniasis, candidiasis and UTI after delivery was $10.5,0.5$ and $3.1 \%$ respectively. The prevalence of trichomoniasis, candidiasis and UTI was $8.3,0.3,3.2 \%$ respectively, in the LNS group, and was similar in the IFA and MMN groups (Table 3). Relative to the IFA group, the risk for trichomoniasis was not significantly different in the SQ-LNS (RR 0.71, 95\% CI 0.47- 1.09) or MMN (RR 0.93, 95\% CI 0.631.37) groups. Similarly, when compared to MMN, the risk for trichomoniasis in the LNS group was not significantly different (RR 0.77, 95\% CI 0.50- 1.18). The risk for UTI was also similar in all 3 study groups (Table 3 ). The risk ratio for candidiasis was not calculated because the prevalence of candidiasis was very low.
Tests of interaction with potential effect modifiers were not significant $(P>0.1)$ for maternal age, maternal parity, socio-economic score, HIV status, anaemia, season at enrolment and study site, but were significant for at least one outcome for gestational age at enrolment, maternal BMI, maternal education and baseline $P$. falciparum parasitaemia. Gestational age at enrolment modified the association between the intervention group and $P$. falciparum parasitaemia by RDT at $32 \mathrm{gw}(P=0.024)$; maternal BMI modified the association between the intervention group and trichomoniasis $(P=0.039)$ : maternal education modified the association between the intervention group and $P$. falciparum by RDT at delivery $(P=0.048)$ and baseline $P$. falciparum parasitaemia modified the association between the intervention group and $P$. falciparum parasitaemia at delivery (by both RDT and PCR, $P=0.058$ and 0.078 respectively). However, there were no statistically significant differences between the intervention groups in any of the strata (Additional file 2: Table S2).

\section{Discussion}

We investigated whether the provision of SQ-LNS during pregnancy affects the occurrence of $P$. falciparum parasitaemia and RTIs among women in a population with generally poor food security. Overall, we found no statistically significant differences in the prevalence of maternal $P$. falciparum parasitaemia and RTIs between the study intervention groups.

The probabilities of bias or random error in this study were minimised by the large sample size, randomised study design, blinding of outcome assessors and inclusion of several $P$. falciparum parasitemia measurements using sensitive detection methods (RDT and PCR) [31, 33]. The success of follow-up was not complete, due to loss of participants during the follow up or missed visits or biological samples, but the proportion of missing data was similar across the study groups and several adjusted analyses gave results similar to the main analysis; hence we believe that the missing data did not significantly bias our conclusions. The excluded participants were,

Table 2 Prevalence of maternal P. falciparum parasitaemia in Malawian women given IFA, MMN and LNS

\begin{tabular}{lllllll}
\hline Outcome & IFA & MMN & LNS & LNS vs. IFA & LNS vs. MMN & MMN vs. IFA \\
\hline $\begin{array}{l}\text { P. falciparum parasitaemia } \\
\text { at } 32 \text { gw (RDT) }\end{array}$ & $12.0(8.7-15.3)(374)^{\mathrm{a}}$ & $9.5(6.6-12.5)(377)$ & $10.9(7.7-14.2)(357)$ & $0.91(0.61-1.36)$ & $1.14(0.74-1.76)$ & $0.79(0.52-1.20)$ \\
$\begin{array}{l}\text { P. falciparum parasitaemia } \\
\text { at } 36 \text { gw (PCR) }\end{array}$ & $10.8(7.5-14.1)(352)$ & $8.5(5.6-11.4)(363)$ & $7.6(4.8-10.4)(355)$ & $0.70(0.44-1.13)$ & $0.89(0.54-1.46)$ & $0.79(0.50-1.24)$ \\
$\begin{array}{l}\text { P. falciparum parasitaemia } \\
\text { at delivery (RDT) }\end{array}$ & $9.1(6.2-12.0)(373)$ & $7.8(5.1-10.5)(372)$ & $8.0(5.3-10.8)(386)$ & $0.88(0.55-1.40)$ & $1.03(0.63-1.67)$ & $0.86(0.53-1.37)$ \\
$\begin{array}{l}\text { P. falciparum parasitaemia } \\
\text { at delivery (PCR) }\end{array}$ & $20.6(16.5-24.7)(369)$ & $22.4(18.0-26.8)(357)$ & $17.6(13.7-21.5)(369)$ & $0.86(0.63-1.15)$ & $0.79(0.59-1.05$ & $1.09(0.82-1.44)$
\end{tabular}

${ }^{a}$ Values are percentages $(95 \% \mathrm{Cls})(\mathrm{n})$ or risk ratios $(95 \% \mathrm{Cls})$. IFA iron folic acid, MMN multiple micronutrients, LNS lipid based nutrient supplements, RDT rapid diagnostic testing, $P C R$ polymerase chain reaction 
Table 3 Prevalence of reproductive tract infections after delivery in Malawian women given IFA, MMN and LNS

\begin{tabular}{lllllll}
\hline Outcome & IFA & MMN & LNS & LNS vs. IFA & LNS vs. MMN & MMN vs. IFA \\
\hline Prevalence of trichomoniasis & $11.6(8.5-14.7)(405)^{\mathrm{a}}$ & $10.8(7.8-13.8)(407)$ & $8.3(5.6-11.0)(398)$ & $0.71(0.47-1.09)$ & $0.77(0.50-1.18)$ & $0.93(0.63-1.37)$ \\
Prevalence of candidiasis & $0.2(-0.2-0.7 \%)(406)$ & $1.0(0.0-1.9)(409)$ & $0.3(-0.2-0.7)(399)$ & - & - & - \\
Prevalence of UTI & $2.2(0.7-3.7)(406)$ & $3.7(1.9-5.6)(405)$ & $3.2(1.5-5.0)(401)$ & $1.46(0.63-3.38)$ & $0.86(0.42-1.82)$ & $1.67(0.74-3.77)$
\end{tabular}

${ }^{a}$ Values are percentages $(95 \% \mathrm{Cls})(\mathrm{n})$ or risk ratios $(95 \% \mathrm{Cls})$. IFA iron folic acid, MMN multiple micronutrients, LNS lipid based nutrient supplements, UTI urinary tract infection

however somewhat different from those included in the analysis in terms of some of their anthropometric, sociodemographic and health characteristics. For these reasons our results may not be fully representative of the target population. Furthermore, the methods that we used to detect vaginal candidiasis, trichomoniasis and bacterial UTI have low to moderate sensitivity [34-36]. This may have led us to underestimate the burden of these conditions in our study population. Finally, the prevalence of each infection (except for $P$. falciparum parasitaemia at $36 \mathrm{gw}$ by PCR) was lower than what we had estimated in our sample size calculation, potentially affecting our power calculations. However, as argued by Feinstein and Concato [37], our point estimates and confidence intervals were consistently in the same direction and sufficiently precise to conclude that SQ-LNS supplementation did not influence the prevalence of maternal $P$. falciparum parasitemia or RTIs in rural Malawi, though we cannot conclusively rule out an effect on the prevalence of candidiasis and UTI.

The prevalence of $P$. falciparum parasitemia at delivery was much higher based on PCR testing compared to RDT among the study women. This finding was not unusual and it was probably due to low parasite density in the study population. The sensitivity of RDT tests varies with parasite density, with lower sensitivity at lower parasite density [38].

The prevalence of $P$. falciparum parasitaemia by RDT continued to decline from enrolment through 32 gestational weeks to delivery in our study population. This finding is unsurprising as similar results were obtained from women who received two doses of IPT-SP in a previous study conducted in the same study area [14] and from elsewhere in sub-Saharan Africa [39]. Assuming no loss to follow-up in our study, we would expect the prevalence of $P$. falciparum parasitaemia in pregnancy to drop in the period following enrolment into the study as the women were exposed to anti-malaria interventions such as IPT-SP and receipt of LLINs as part of the ante-natal care package.

Little is known about the effect of SQ-LNS on maternal health or morbidity. Other reports from the iLiNSDYAD trial suggested that SQ-LNS and MMN may have increased the risk of maternal dental infections [40] on one hand but seemed to have had no effect on the development of maternal anti-malarial antibodies [41] on the other hand. Previous in vitro studies have suggested that EFAs and their derivatives such as arachidonic acid and docosahexaenoic acid may have toxic effects on $P$. falciparum [42], T. vaginalis [43] and C. albicans [27] via different mechanisms, hence our expectation that providing SQ-LNS which contained EFAs would reduce the prevalence of infections caused by these organisms. The apparent lack of benefit from the EFAs in this study could have happened because the study women were not deficient in EFAs to begin with. A recently published trial conducted in the same study area as our study found that the levels of breast milk arachidonic acid and docosahexaenoic acid were above average levels [44] contrary to our earlier assumptions based on previous studies in sub-Saharan Africa [45]. Alternatively, the absence of influence of SQ-LNS on maternal plasma composition of EFAs (Oaks et al., in press) could explain our observation of lack of impact.

A previous study that provided combined vitamin A and zinc supplementation to children in Ghana led to a reduction in the risk of clinical malaria episodes [46] possibly due to the synergistic effects of vitamin A and zinc on immune function [47]. Contrary to our expectation based on these previous studies, SQ-LNS and MMN which contained both vitamin A and zinc were not associated with a lower risk of $P$. falciparum parasitaemia. The lack of treatment response despite the provision of adequate doses of vitamin $\mathrm{A}$ and zinc in MMN and SQ-LNS and reasonable adherence to supplementation [24] could have been due to dietary factors inhibiting zinc absorption [48] or haemodilution in the latter half of pregnancy lowering levels of retinol binding protein resulting in functional vitamin A deficiency [49].

\section{Conclusion}

Taken together, the study findings do not support our hypothesis that the provision of antenatal SQ-LNS would reduce the prevalence of $P$. falciparum parasitaemia during pregnancy and the prevalence of RTIs after delivery compared to supplementation with MMN or IFA. Whether or not a target population is deficient in EFAs might be an important factor in determining whether SQ-LNS provision to pregnant women can 
influence the occurrence of maternal infections. Use of more sensitive diagnostic methods such as culture, antigen or PCR tests for the detection of RTIs and UTI may improve outcome ascertainment in future studies. Further clinical trials supplementing single nutrients (EFAs, vitamin A or zinc) may be crucial in isolating the impact of each nutrient on maternal infections.

\section{Additional files}

Additional file 1: Table S1. Nutrient contents of the dietary supplements provided in the iLiNS-DYAD-M Trial. (DOCX $17 \mathrm{~kb}$ )

Additional file 2: Table S2. Effect Modification - Malaria Parasitaemia and Trichomoniasis by Intervention Group, Stratified Analysis. (DOCX 19 kb)

\section{Abbreviations}

BMI: Body-mass-index; Cls: Confidence Intervals; EFAs: Essential fatty acids; gw: Gestation weeks; IFA: Iron and folic acid; IPT-SP: Intermittent preventive treatment with sulphadoxine pyrimethamine; LBW: Low birth weight; LLINs: Long-lasting insecticide treated nets; MMN: Multiple micronutrients; PCR: Polymerase chain reaction; RDT: Rapid diagnostic testing; RRs: Risk ratios; RTIs: Reproductive tract infections; SQ-LNS: Small-quantity lipid-based nutrient supplements; UTI: Urinary tract infection

\section{Funding}

This publication is based on research funded in part by the Office of Health, Infectious Diseases, and Nutrition, Bureau for Global Health, U.S. Agency for International Development (USAID) under terms of Cooperative Agreement No. AID-OAA-A-12-00005, through the Food and Nutrition Technical Assistance III Project (FANTA), managed by FHI 360, with additional funding from a grant to the University of California, Davis by the Bill \& Melinda Gates Foundation. For data management and statistical analysis, the team received additional support in grants from the Academy of Finland (grant 252075) and the Medical Research Fund of Tampere University Hospital (grant 9 M004). The findings and conclusions contained within are those of the authors and do not necessarily reflect positions or policies of the United States Government, USAID, the Bill \& Melinda Gates Foundation, or the other funders. The funders had no role in study design, data collection and analysis, decision to publish, or preparation of the manuscript.

\section{Availability of data and materials}

The datasets supporting the conclusions of this article are included within the article (and its additional files).

\section{Authors' contributions}

The authors' contributions were as follows: PA, UA, KGD, and KM: designed the research; $P A, U A, M N, A G$ and $K M$ : conducted the research; $M N$ and JM: analyzed data; $\mathrm{MN}$ : wrote the manuscript, with critical input and comments from all other authors; and MN and KM: had primary responsibility for final content. All authors read and approved the final manuscript. The findings and conclusions contained within the article are those of the authors and do not necessarily reflect positions or policies of the Bill \& Melinda Gates Foundation, USAID, the US government, or the other funders.

\section{Competing interests}

The authors declare that they have no competing interests.

\section{Consent for publication}

Not applicable.

\section{Ethics approval and consent to participate}

The study protocol for the iLiNS-DYAD-M trial was approved by the College of Medicine Research and Ethics Committee, University of Malawi and the Ethics Committee of Pirkanmaa Hospital District, Finland. Informed consent was obtained from all the study participants prior to conducting any study procedures.

\section{Author details}

'University of Tampere School of Medicine, University of Tampere, Arvo building, Fl-33014 Tampere, Finland. ${ }^{2}$ Department of Pediatrics, Tampere University Hospital, Fl-33521 Tampere, Finland. ${ }^{3}$ Department of Nutrition, University of California, One Shields Ave., Davis, CA 95616-8669, USA. ${ }^{4}$ School of Public Health and Family Medicine, College of Medicine, Mahatma Gandhi Road, Blantyre, Malawi. ${ }^{5}$ Department of Medicine at the Peter Doherty Institute, 792 Elizabeth Street, Melbourne, VIC 3000, Australia. ${ }^{6}$ Division of Infectious Diseases and International Health, Duke University Medical Center, Box 102359 DUMC, Durham, NC 27705, USA. ${ }^{7}$ Department of Epidemiology, University of North Carolina, Chapel Hill, NC 27599-7435, USA.

Received: 6 May 2016 Accepted: 29 December 2016

Published online: 17 January 2017

\section{References}

1. Chico RM, Mayaud P, Ariti C, Mabey D, Ronsmans C, Chandramohan D. Prevalence of malaria and sexually transmitted and reproductive tract infections in pregnancy in sub-Saharan Africa: a systematic review. JAMA. 2012;307(19):2079-86.

2. Steketee RW, Nahlen BL, Parise ME, Menendez C. The burden of malaria in pregnancy in malaria-endemic areas. Am J Trop Med Hyg. 2001;64(1-2 Suppl):28-35.

3. Steketee RW, Wirima JJ, Hightower AW, Slutsker L, Heymann DL, Breman JG. The effect of malaria and malaria prevention in pregnancy on offspring birthweight, prematurity, and intrauterine growth retardation in rural Malawi. Am J Trop Med Hyg. 1996;55(1 Suppl):33-41.

4. Silver BJ, Guy RJ, Kaldor JM, Jamil MS, Rumbold AR. Trichomonas vaginalis as a cause of perinatal morbidity: a systematic review and meta-analysis. Sex Transm Dis. 2014;41(6):369-76.

5. Kiss H, Petricevic L, Husslein P. Prospective randomised controlled trial of an infection screening programme to reduce the rate of preterm delivery. BMJ. 2004;329(7462):371.

6. Schieve LA, Handler A, Hershow R, Persky V, Davis F. Urinary tract infection during pregnancy: its association with maternal morbidity and perinatal outcome. Obstet Gynecol Surv. 1994;49(9):596-7.

7. Roberts $\mathrm{CL}$, Algert CS, Rickard KL, Morris JM. Treatment of vaginal candidiasis for the prevention of preterm birth: a systematic review and meta-analysis. Syst Rev. 2015;4:31.

8. Kurewa NE, Mapingure MP, Munjoma MW, Chirenje MZ, Rusakaniko S, StrayPedersen B. The burden and risk factors of Sexually Transmitted Infections and Reproductive Tract Infections among pregnant women in Zimbabwe. BMC Infect Dis. 2010;10:127.

9. Nwosu CO, Djieyep NA. Candidiasis and trichomoniasis among pregnant women in a rural community in the semi-arid zone, north-eastern Nigeria. West Afr J Med. 2007;26(1):17-9.

10. Moster D, Lie RT, Markestad T. Long-term medical and social consequences of preterm birth. N Engl J Med. 2008;359(3):262-73.

11. McCormick MC. The contribution of low birth weight to infant mortality and childhood morbidity. N Engl J Med. 1985;312(2):82-90.

12. Hansen BM. Long-term consequences of pre-term birth. Pediatr Res. 2011;70(5):19.

13. Feng G, Simpson JA, Chaluluka E, Molyneux ME, Rogerson SJ. Decreasing burden of malaria in pregnancy in Malawian women and its relationship to use of intermittent preventive therapy or bed nets. PLoS One. 2010;5(8):e12012.

14. Luntamo M, Kulmala T, Mbewe B, Cheung YB, Maleta K, Ashorn P. Effect of repeated treatment of pregnant women with sulfadoxine-pyrimethamine and azithromycin on preterm delivery in Malawi: a randomized controlled trial. Am J Trop Med Hyg. 2010;83(6):1212-20.

15. World Health Organisation. Malaria in Pregnancy. 2015. http://www.who.int/ malaria/areas/high_risk_groups/pregnancy/en/. Accessed 18 Nov 2015.

16. Sangkomkamhang US, Lumbiganon P, Prasertcharoensuk W, Laopaiboon M. Antenatal lower genital tract infection screening and treatment programs for preventing preterm delivery. Cochrane Database Syst Rev. 2015;2:006178.

17. Wondji CS, Coleman M, Kleinschmidt I, Mzilahowa T, Irving H, Ndula M, et al. Impact of pyrethroid resistance on operational malaria control in Malawi. Proc Natl Acad Sci U S A. 2012;109(47):19063-70.

18. Artimovich E, Schneider K, Taylor TE, Kublin JG, Dzinjalamala FK, Escalante $A A$, et al. Persistence of sulfadoxine-pyrimethamine resistance despite reduction of drug pressure in Malawi. J Infect Dis. 2015;212(5):694-701. 
19. Roca I, Akova M, Baquero F, Carlet J, Cavaleri M, Coenen S, et al. The global threat of antimicrobial resistance: science for intervention. New Microbes New Infect. 2015;6:22-9.

20. Arimond $\mathrm{M}$, Zeilani M, Jungjohann S, Brown KH, Ashorn $\mathrm{P}$, Allen LH, et al. Considerations in developing lipid-based nutrient supplements for prevention of undernutrition: experience from the International Lipid-Based Nutrient Supplements (iLiNS) Project. Matern Child Nutr. 2015;11 Suppl 4:31-61.

21. Adu-Afarwuah S, Lartey A, Okronipa H, Ashorn P, Zeilani M, Peerson JM, et al. Lipid-based nutrient supplement increases the birth size of infants of primiparous women in Ghana. Am J Clin Nutr. 2015;101(4):835-46.

22. Mridha MK, Matias SL, Chaparro CM, Paul RR, Hussain S, Vosti SA, et al. Lipid-based nutrient supplements for pregnant women reduce newborn stunting in a cluster-randomized controlled effectiveness trial in Bangladesh. Am J Clin Nutr. 2016;103(1):236-49.

23. Huybregts L, Roberfroid D, Lanou H, Menten J, Meda N, Van Camp J, et al. Prenatal food supplementation fortified with multiple micronutrients increases birth length: a randomized controlled trial in rural Burkina Faso. Am J Clin Nutr. 2009;90(6):1593-600.

24. Ashorn P, Alho L, Ashorn U, Cheung YB, Dewey KG, Harjunmaa U, et al. The impact of lipid-based nutrient supplement provision to pregnant women on newborn size in rural Malawi: a randomized controlled trial. Am J Clin Nutr. 2015;101(2):387-97.

25. Kumaratilake LM, Robinson BS, Ferrante A, Poulos A. Antimalarial properties of $n-3$ and $n-6$ polyunsaturated fatty acids: in vitro effects on Plasmodium falciparum and in vivo effects on P. berghei. J Clin Invest. 1992;89(3):961-7.

26. Korosh T, Jordan KD, Wu JS, Yarlett N, Upmacis RK. Eicosapentaenoic acid modulates Trichomonas vaginalis activity. J Eukaryot Microbiol. 2016;63(2):153-61.

27. Thibane VS, Ells R, Hugo A, Albertyn J, van Rensburg WJ, Van Wyk PW, et al. Polyunsaturated fatty acids cause apoptosis in C. albicans and C. dubliniensis biofilms. Biochim Biophys Acta. 2012;1820(10):1463-8.

28. Institute of Medicine. Dietary reference intakes for vitamin A, vitamin K, Arsenic, Boron, Chromium, Copper, lodine, Iron, Manganese, Molybdenum, Nickel, Silicon, Vanadium and Zinc. 2001.

29. Zhou SJ, Gibson RA, Crowther CA, Makrides M. Should we lower the dose of iron when treating anaemia in pregnancy? A randomized dose-response trial. Eur J Clin Nutr. 2009:63(2):183-90.

30. World Health Organisation. Report of the technical expert group meeting on intermittent preventive treatment in pregnancy (IPTp). Geneva, 2007 July 11-13. 2008

31. Rantala AM, Taylor SM, Trottman PA, Luntamo M, Mbewe B, Maleta K, et al. Comparison of real-time PCR and microscopy for malaria parasite detection in Malawian pregnant women. Malar J. 2010;9:269.

32. Mambatta AK, Jayarajan J, Rashme VL, Harini S, Menon S, Kuppusamy J. Reliability of dipstick assay in predicting urinary tract infection. J Family Med Prim Care. 2015;4(2):265-8.

33. World Health Organisation. Malaria rapid diagnostic test performance results of WHO product testing of malaria RDTs: round 3 (2010-2011). 2011. http:// www.who.int/tdr/publications/tdr-research-publications/rdt_round3/en/. Accessed 21 Oct 2016

34. Chatwani AJ, Mehta R, Hassan S, Rahimi S, Jeronis S, Dandolu V. Rapid testing for vaginal yeast detection: a prospective study. Am J Obstet Gynecol. 2007;196(4):309. e1-309.e4

35. Radonjic IV, Dzamic AM, Mitrovic SM, Arsic Arsenijevic VS, Popadic DM, Kranjcic Zec IF. Diagnosis of Trichomonas vaginalis infection: the sensitivities and specificities of microscopy, culture and PCR assay. Eur J Obstet Gynecol Reprod Biol. 2006;126(1):116-20.

36. Rogozinska E, Formina S, Zamora J, Mignini L, Khan KS. Accuracy of onsite tests to detect asymptomatic bacteriuria in pregnancy: a systematic review and meta-analysis. Obstet Gynecol. 2016;128(3):495-503.

37. Feinstein AR, Concato J. The quest for "power": contradictory hypotheses and inflated sample sizes. J Clin Epidemiol. 1998;51(7):537-45.

38. Kattenberg JH, Tahita CM, Versteeg IA, Tinto H, Traore Coulibaly M, D'Alessandro $U$, et al. Evaluation of antigen detection tests, microscopy, and polymerase chain reaction for diagnosis of malaria in peripheral blood in asymptomatic pregnant women in Nanoro, Burkina Faso. Am J Trop Med Hyg. 2012;87(2):251-6.

39. Kyabayinze DJ, Zongo I, Cunningham J, Gatton M, Angutoko P, Ategeka J, et al. HRP2 and pLDH-based rapid diagnostic tests, expert microscopy, and PCR for detection of malaria infection during pregnancy and at delivery in areas of varied transmission: a prospective cohort study in Burkina Faso and Uganda. PLoS One. 2016;11(7):e0156954.
40. Harjunmaa U, Jarnstedt J, Dewey KG, Ashorn U, Maleta K, Vosti SA, et al. Nutrient supplementation may adversely affect maternal oral health - a randomised controlled trial in rural Malawi. Matern Child Nutr. 2016;12(1):99-110.

41. Chandrasiri UP, Fowkes FJ, Richards JS, Langer C, Fan YM, Taylor SM, et al. The impact of lipid-based nutrient supplementation on anti-malarial antibodies in pregnant women in a randomized controlled trial. Malar J. 2015;14:193.

42. Melariri P, Campbell W, Etusim P, Smith P. In vitro and in vivo antimalarial activity of linolenic and linoleic acids and their methyl esters. Adv Stud Biol. 2012;4(7):333-49.

43. Korosh T, Jordan KD, Wu JS, Yarlett N, Upmacis RK. Eicosapentaenoic Acid Modulates Trichomonas vaginalis Activity. J Eukaryot Microbiol. 2016;63(2):153-61.

44. Jimenez EY, Mangani C, Ashorn P, Harris WS, Maleta K, Dewey KG. Breast milk from women living near Lake Malawi is high in docosahexaenoic acid and arachidonic acid. Prostaglandins Leukot Essent Fatty Acids. 2015;95:71-8.

45. Michaelsen KF, Dewey KG, Perez-Exposito AB, Nurhasan M, Lauritzen L, Roos N. Food sources and intake of n-6 and n-3 fatty acids in low-income countries with emphasis on infants, young children (6-24 months), and pregnant and lactating women. Matern Child Nutr. 2011;7 Suppl 2:124-40.

46. Owusu-Agyei S, Newton S, Mahama E, Febir LG, Ali M, Adjei K, et al. Impact of vitamin A with zinc supplementation on malaria morbidity in Ghana. Nutr J. 2013;12:131.

47. Christian P, West Jr KP. Interactions between zinc and vitamin A: an update. Am J Clin Nutr. 1998;68(2 Suppl):435S-41.

48. Lonnerdal B. Dietary factors influencing zinc absorption. J Nutr. 2000;130(5S Suppl):1378S-83.

49. Sapin V, Alexandre MC, Chaib S, Bournazeau JA, Sauvant $P$, Borel $P$, et al. Effect of vitamin A status at the end of term pregnancy on the saturation of retinol binding protein with retinol. Am J Clin Nutr. 2000;71(2):537-43.

\section{Submit your next manuscript to BioMed Central and we will help you at every step:}

- We accept pre-submission inquiries

- Our selector tool helps you to find the most relevant journal

- We provide round the clock customer support

- Convenient online submission

- Thorough peer review

- Inclusion in PubMed and all major indexing services

- Maximum visibility for your research

Submit your manuscript at www.biomedcentral.com/submit
C BioMed Central 